\title{
Measurement-induced entanglement transitions in many-body localized systems
}

\author{
Oliver Lunt $\odot^{*}$ and Arijeet Pal ${ }^{\dagger}$ \\ Department of Physics, University College London, Gower Street, London WC1E 6BT, United Kingdom
}

(Received 2 June 2020; accepted 11 September 2020; published 14 October 2020)

\begin{abstract}
The resilience of quantum entanglement to a classicality-inducing environment is tied to fundamental aspects of quantum many-body systems. The dynamics of entanglement has recently been studied in the context of measurement-induced entanglement transitions, where the steady-state entanglement collapses from a volume law to an area law at a critical measurement probability $p_{c}$. Interestingly, there is a distinction in the value of $p_{c}$ depending on how well the underlying unitary dynamics scramble quantum information. For strongly chaotic systems, $p_{c}>0$, whereas for weakly chaotic systems, such as integrable models, $p_{c}=0$. In this work, we investigate these measurement-induced entanglement transitions in a system where the underlying unitary dynamics are many-body localized (MBL). We demonstrate that the emergent integrability in an MBL system implies a qualitative difference in the nature of the measurement-induced transition depending on the measurement basis, with $p_{c}>0$ when the measurement basis is scrambled and $p_{c}=0$ when it is not. This feature is not found in Haar-random circuit models, where all local operators are scrambled in time. When the transition occurs at $p_{c}>0$, we use finite-size scaling to obtain the critical exponent $v=1.3(2)$, close to the value for (2+0)-dimensional percolation. We also find a dynamical critical exponent of $z=0.98(4)$ and logarithmic scaling of the Rényi entropies at criticality, suggesting an underlying conformal symmetry at the critical point. This work further demonstrates how the nature of the measurement-induced entanglement transition depends on the scrambling nature of the underlying unitary dynamics. This leads to further questions on the control and simulation of entangled quantum states by measurements in open quantum systems.
\end{abstract}

DOI: 10.1103/PhysRevResearch.2.043072

\section{INTRODUCTION}

The environment plays a profound role in the dynamics of quantum systems. The loss of coherence of the quantum system is facilitated by the environment which ultimately leads to the emergence of classical behavior at the macroscopic scale [1]. While the dynamics of an isolated quantum system is unitary, the coupling to the environment renders it nonunitary. Dynamics of quantum entanglement due to nonunitary dynamics is therefore crucial for the understanding of the quantum to classical crossover. A particular framework in which to explore nonunitary dynamics is in the context of quantum measurements. The influence of measurements has shed new light on the dynamics of information in quantum many-body systems and provided potential mechanisms for purification [2,3] and quantum error correction [4]. Exciting experimental developments over the past decade in quantum control and simulation of few and many-body systems have shed light on the interplay between measurement, entanglement, and decoherence [5-8].

\footnotetext{
*oliver.lunt.17@ucl.ac.uk

†a.pal@ucl.ac.uk

Published by the American Physical Society under the terms of the Creative Commons Attribution 4.0 International license. Further distribution of this work must maintain attribution to the author(s) and the published article's title, journal citation, and DOI.
}

Measurement-induced dynamics has led to the unravelling of a new class of entanglement phase transitions driven by quantum measurements [9-31] in random unitary circuits, which have served as effective models for quantum chaos and thermalization. The study of these transitions has also led to new insights into emergent quantum error correction in many-body quantum systems [24,32,33], and to efficient classical algorithms for the simulation of random shallow $(2+1)$-dimensional $(2+1 \mathrm{D})$ quantum circuits [34]. The measurements drive the system into a steady state which either satisfies a volume law for entanglement or an area law, depending on how frequently the quantum degrees of freedom are being measured. The transition only occurs in an individual quantum trajectory conditioned on the measurements: averaging over the measurements washes out the distinct entanglement structure in the steady states in the two phases. Although in these toy models of the transition the measurements are projective, analogous transitions are also realized in continuously measured systems. These $1+1 \mathrm{D}$ quantum circuits can be mapped to a $2+0 \mathrm{D}$ classical statistical mechanical model, which in the limit of large local Hilbert space dimension maps to a percolation model [10,11,13,22], explaining the emergence of criticality at the transition, and critical exponents which are mostly those of $2+0 \mathrm{D}$ percolation.

Although not easily tractable, the study of the effect of measurements on Hamiltonian dynamics has potentially direct relevance to experiments. It is experimentally viable to realize nonintegrable quantum Hamiltonians whose effective dynamics is described by random unitary circuits [35-38]. 
(a)

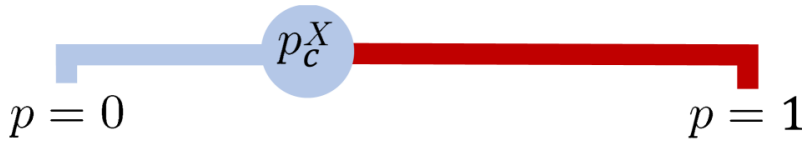

$p=0$

(b)

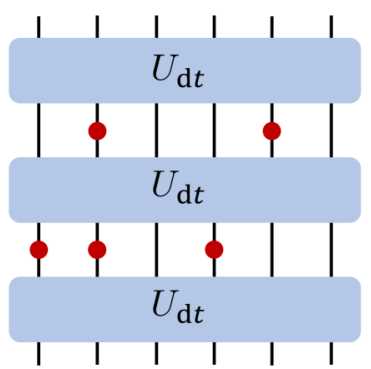

(c)

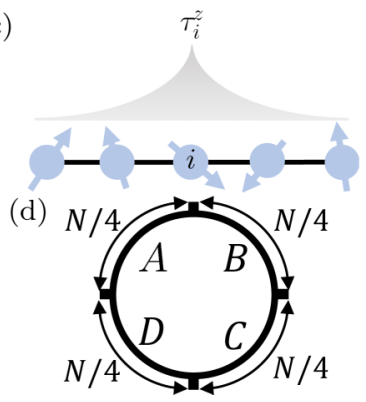

FIG. 1. (a) Critical properties of the transition depend on the measurement basis. Measurements in the $X$-basis result in a transition from volume-law to area-law entanglement at nonzero $p=p_{c}^{X}>0$, similar to chaotic systems, whereas measurements in the $Z$-basis result in area-law entanglement for any nonzero $p$, similar to integrable systems. (b) Each stage of the dynamics consists of two steps. First the system is evolved in time with the unitary $U_{\mathrm{d} t}=\exp (-i H \mathrm{~d} t)$, with $H$ given in Eq. (1), and then for each spin we projectively measure in a tensor-product basis with probability $p$ (measurements are represented by the red dots). (c) A schematic of the typical support of an " $l$-bit" operator, localized at site $i$, in the fully MBL phase. (d) A depiction of the geometry used to calculate the tripartite information $I_{3}(A: B: C)$.

Systems whose dynamics are governed by an interacting Hamiltonian exhibit two classes of dynamics. At one end are chaotic systems, which can variously be characterized as those systems which spread entanglement ballistically and saturate to volume-law steady state entanglement, complying with equilibrium thermodynamics [39]. At the other end are manybody localized (MBL) systems, which violate equilibrium thermodynamics [40-46], and can similarly be characterized by their logarithmic spreading of entanglement in time and also saturate to volume-law steady-state entanglement, albeit lower than the thermodynamic entropy density [47-51].

In this work we study the dynamics of an MBL Hamiltonian subjected to projective measurements. Many-body localized systems are characterized by a complete set of quasilocal operators, also known as "l-bits," which are conserved under the dynamics of the system's Hamiltonian [see Fig. 1(c)] [49,50,52,53]. Due to this robust integrability, one might guess that the measurement-induced entanglement transition in MBL systems is similar to that in integrable systems. Although measurements in MBL and disorder-free integrable models share certain similarities, here we show that measurement-induced transitions performed in certain bases [see Fig. 1(a)] can have distinct properties.

To illustrate this point, recall that in a chaotic quantum system local operators spread ballistically, resulting in the rapid scrambling of information with time which can be diagnosed through the decay of out-of-time-order correlators (OTOCs). The implication of this for the measurement-induced

entanglement transition is that in chaotic systems the choice of measurement basis does not matter, provided it is local (i.e., close to a tensor product basis). However, in an MBL system, only operators without any overlap with the $l$-bits are totally scrambled with their out-of-time ordered correlators decaying to zero. For example, the late-time limit of the disorder-averaged OTOC of an operator is set by its overlap with the local integrals of motion of the MBL system [54-59]. For the model described by Eq. (1), in the strong-disorder limit $W \gg 1$ the $l$-bits $\left\{\tau_{i}^{z}\right\}$ are close to the local spin operators $\left\{S_{i}^{z}\right\}$, dressed by exponentially decaying tails [60-64], as shown in Fig. 1(c). The $l$-bits are related to the physical spins by a quasilocal unitary $U$ via $\tau_{i}^{z}=U S_{i}^{z} U^{\dagger}$, with $U \rightarrow \mathbb{1}$ in the strong-disorder limit. This means that the overlap of an operator $\mathcal{O}$ with an $l$-bit $\tau_{i}^{z}$ is given by $\operatorname{Tr}\left[\mathcal{O} \tau_{i}^{z}\right]=\operatorname{Tr}\left[\mathcal{O} S_{i}^{z}\right]+$ $\cdots$, where the dots indicate terms which vanish in the limit $W \rightarrow \infty$. As a result, the operator $S_{i}^{x}$ is scrambled by the MBL system, whereas the operator $S_{i}^{z}$ remains approximately localized.

This has the consequence that there is a qualitative difference between the nature of the measurement-induced entanglement transition in an MBL system depending on whether the measurements are performed in the basis of $S_{i}^{x}$ eigenstates ( $X$ basis) or the basis of $S_{i}^{z}$ eigenstates ( $Z$ basis), as shown in Fig. 1(a). With measurements in the $X$ basis, the transition from volume to area-law entanglement occurs at a nonzero measurement probability $p_{c}^{X}>0$, similar to previously studied chaotic systems [9-16,18,19,21-25,32,33]. On the other hand, with measurements in the $Z$ basis, the volume law is destroyed for any nonzero $p$, similar to previously studied integrable systems $[12,17,20]$.

This difference can also be related to the interplay between measurements and the phenomenology of entanglement growth in MBL systems. As we discuss in Sec. IIB, in a measurement-free MBL system, the steady-state entanglement is governed by the diagonal entropy, which is constant under Hamiltonian time evolution. Here we show that measurements induce dynamics in the diagonal entropy which qualitatively differ between $X$-basis measurements and $Z$ basis measurements, where $X$-basis measurements tend to maximize the diagonal entropy while $Z$-basis measurements tend to minimize it. This then drives the steady state to volume-law or area-law entanglement, depending on the measurement basis.

In a recent work [65], the authors investigated the dynamics of a finite-range $l$-bit model of MBL under local Clifford dynamics. We analyze the full $l$-bit Hamiltonian, which includes infinite-range interactions between $l$-bits. It also provides a picture of the measurement-induced entanglement transition in a fully realistic model of MBL and our results are consistent with results in Ref. [65], where they can be compared.

\section{MODEL}

We study a standard model of MBL, the disordered spin- $\frac{1}{2}$ Heisenberg chain, with a Hamiltonian given by

$$
H=J \sum_{\langle i j\rangle} \mathbf{S}_{i} \cdot \mathbf{S}_{j}+\sum_{i=1}^{N} h_{i} S_{i}^{z},
$$


where the $h_{i}$ are random variables drawn from the uniform distribution on $[-h, h]$ and the double sum runs over nearest neighbors. We use periodic boundary conditions unless stated otherwise. For sufficiently large disorder strength $W \equiv h / J$ this model is many-body localized. Early studies of MBL based on exact diagonalization estimated the critical disorder strength $W_{c}$ to be around $W_{c} \approx 3.6[41,66]$, though more recent studies have argued that $W_{c}$ has significant finite-size corrections, and have suggested a figure of $W_{c} \approx 5$ [67]. In this paper we are interested in the region well into the localized phase, and so will take $W=10$, large enough to avoid issues with finite-size drifts of $W_{c}$.

To study the effects of measurements on the entangling properties of MBL dynamics, we consider a repeated twostage protocol, as illustrated in Fig. 1(b). Starting from an initial Haar-random product state, we apply unitary dynamics generated by the Hamiltonian for some time $\mathrm{d} t$, i.e., $|\psi\rangle \rightarrow$ $U_{\mathrm{d} t}|\psi\rangle$, where $U_{\mathrm{d} t}=\exp (-i H \mathrm{~d} t)$. Unless otherwise specified we take $\mathrm{d} t=1$ in units of $J$, independent of $N$, to make the situation comparable with random local circuit models. Then for each spin we projectively measure in a local tensor-product basis with probability $p$, where $p$ can be interpreted as the density of measurements in space-time. The measurement outcomes are chosen according to the Born rule. To implement a measurement on spin $i$ with outcome $\lambda$, the state is updated via the usual rule $|\psi\rangle \rightarrow P_{i}(\lambda)|\psi\rangle / \| P_{i}(\lambda)|\psi\rangle \|_{2}$, where $P_{i}(\lambda)=|\lambda\rangle\left\langle\left.\lambda\right|_{i}\right.$ is the projector onto the eigenspace associated with the measurement outcome $\lambda$. This process is repeated until the entanglement entropies reach a steady state.

\section{A. Transition diagnostics}

To study the measurement-induced entanglement transition in this MBL system, we performed the time evolution using exact diagonalization. We made use of the fact that the Hamiltonian $H$ in Eq. (1) conserves total $S^{z}$, so in a basis ordered by total $S^{z}, H$ is block-diagonal and one can diagonalize the blocks separately.

To characterize the transition we focused on two quantities: the von Neumann entropy $S_{X}=-\operatorname{Tr}\left[\rho_{X} \ln \rho_{X}\right]$ and the tripartite information

$$
I_{3}(A: B: C)=I(A: B)+I(A: C)-I(A: B C),
$$

where $I(A: B)=S_{A}+S_{B}-S_{A B}$ is the mutual information. We calculate $I_{3}(A: B: C) \equiv I_{3}$ for the geometry shown in Fig. 1(d). It is easy to show that, for a system partitioned into four subsystems, the tripartite information of any three of the subsystems does not depend on the choice of subsystems, so once the partitioning is fixed there is no ambiguity in calling this quantity $I_{3}$.

As discussed in Refs. [18,33], the advantage of using the tripartite information here is that it avoids any $\log N$ divergences in the entanglement entropy at criticality, which have been observed in hybrid Haar-random circuit models [18]. Instead, $I_{3}$ is expected to scale as $I_{3} \propto-N$ in the volume-law phase, reach an $O(1)$ constant at criticality, and then vanish in the area-law phase. This is especially important given the limited system sizes accessible by exact diagonalization.

Throughout this paper we will mostly focus on the von Neumann entropy and quantities defined in terms of it.
However, one can also consider the transitions in the broader family of Rényi entropies, defined as

$$
S_{n}(\rho)=\frac{1}{1-n} \ln \operatorname{Tr}\left[\rho^{n}\right] .
$$

The Rényi- $n$ entropy tends to the von Neumann entropy in the limit $n \rightarrow 1$. For $n>1$, there is the inequality $S_{\infty} \leqslant S_{n} \leqslant$ $\frac{n}{n-1} S_{\infty}$, which implies that all Rényi- $n$ entropies with $n>1$ must have the same scaling with system size. We consider the transition in the Rényi entropies in Sec. IV.

\section{B. Diagonal entropy}

The qualitative difference in the dynamics of the entanglement entropies with $Z$ - or $X$-basis measurements can also be related to the interplay of measurements with the phenomenology of entanglement growth in MBL systems. In the absence of measurements, the steady-state entanglement of an MBL system is set by the diagonal entropy of the initial state in the energy eigenbasis [47-51], $S(t \rightarrow \infty) \propto S_{\text {diag }}(|\psi(0)\rangle)$, with

$$
S_{\text {diag }}(|\psi(0)\rangle)=-\sum_{i} p_{i} \ln p_{i}, \quad p_{i}=\left|\left\langle E_{i} \mid \psi(0)\right\rangle\right|^{2},
$$

where the $\left\{\left|E_{i}\right\rangle\right\}$ are the eigenstates of $H$. This is a result of dephasing between $l$-bits. For many classes of initial states, such as random product states, the average diagonal entropy is extensive in the strong disorder regime, thus giving rise to volume-law steady-state entanglement in MBL systems.

It is worth noting that $S_{\text {diag }}$ is constant under time evolution by $H$, since it depends only on the magnitude of the amplitudes $\left\langle E_{i} \mid \psi(t)\right\rangle$, which merely pick up a phase. However, once measurements are introduced, the diagonal entropy is no longer preserved in time. Instead, the measurements drive the diagonal entropy to a new steady state, typically at an exponential rate $\lambda \approx p$. The choice of measurement basis determines the nature of this new steady state. In the strong disorder limit, the eigenstates of $H$ are close to product states of $S_{i}^{z}$ eigenstates. Thus measurements in the $Z$ basis tend to drive the diagonal entropy close to zero (see Fig. 4), whereas measurements in the $X$ basis tend to drive it close to its maximum value (see Fig. 6).

\section{ENTANGLEMENT DYNAMICS WITH Z-BASIS MEASUREMENTS}

In this section we focus on unitary MBL dynamics interspersed with random projective measurements in the $Z$ basis, and demonstrate the fact that there is a qualitative difference once measurements are introduced with any arbitrarily small probability $p$. That this transition occurs at $p_{c}^{Z}=0$ is similar to previously studied integrable systems $[12,17,20]$, which is consistent with the picture of $\mathrm{MBL}$ as a form of quasiintegrability robust to local perturbations.

Figure 2 shows a comparison of the dynamics of the tripartite information $I_{3}$ between $p=0$ and $p>0$. For $p=0, I_{3}$ grows in magnitude logarithmically in time before saturating to a volume law, similar to the well-known behavior of the half-chain entanglement entropy. On the other hand, for $p>0$ the tripartite information initially grows at early times while the diagonal entropy is still large, reaching an area-law peak 

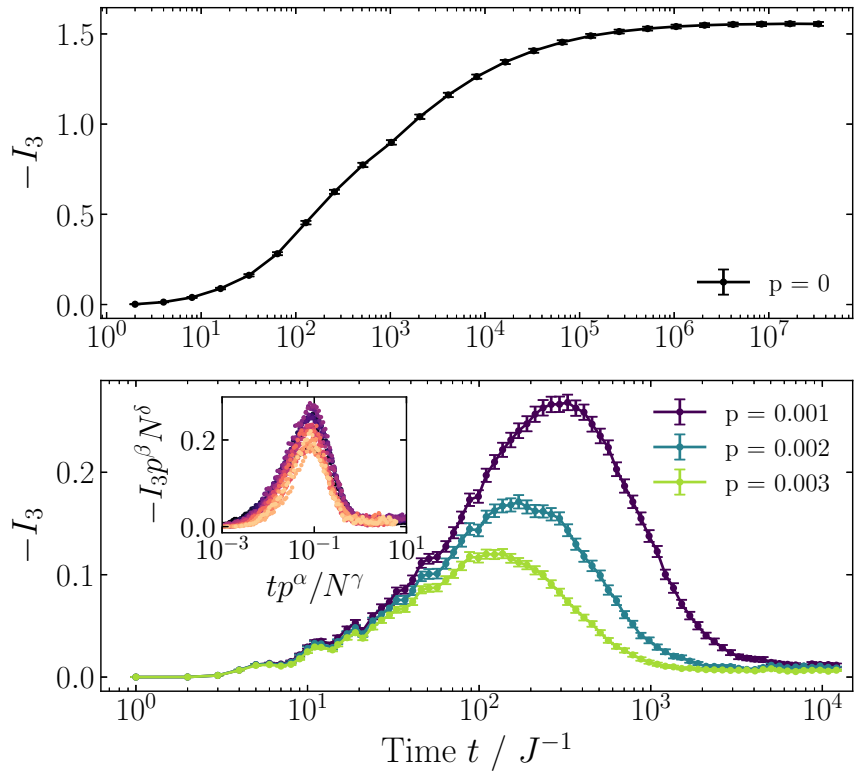

FIG. 2. Comparison of the dynamics of the tripartite information $I_{3}$ for $p=0$ and $p>0$ with $Z$-basis measurements, with $N=12$. For $p=0, I_{3}$ grows logarithmically in time before saturating to a volume law. For $p>0, I_{3}$ initially grows in time, reaching an area-law peak, before decaying to a constant which decreases with system size. The inset shows that the dynamics can be reasonably well described by the scaling form $-I_{3}(t, p, N)=$ $F\left[t p^{\alpha} / N^{\gamma}\right] / p^{\beta} N^{\delta}$, where $F$ is a single-parameter scaling function and $\alpha=0.77, \beta=0.80, \gamma=1.14$, and $\delta=2.19$. The inset data correspond to 20 separate time series, with $N=12,16$ and $0.0005 \leqslant p \leqslant 0.005$.

[see Fig. 3(b)], but eventually the measurements dominate and $I_{3}$ decays to a smaller area law. This area-law peak also appears in the Bell pair model of Ref. [12], but there it occurs at an $O(1)$ time $t_{\text {peak }} \sim 1 / p$. In this system, the peak time does still scale as $1 / p$, but it also increases with system size [see

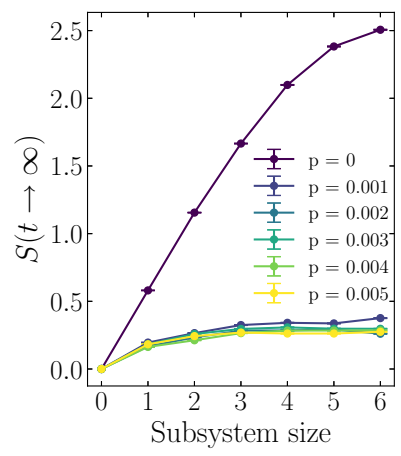

(a)

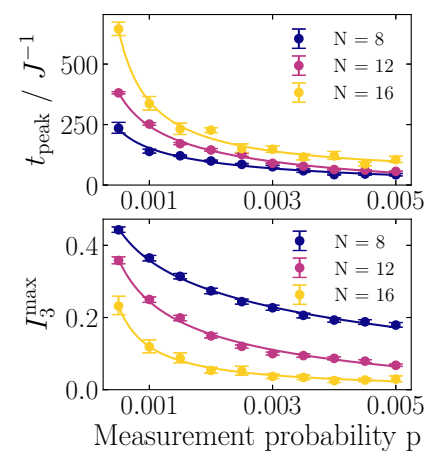

(b)
FIG. 3. (a) Scaling of the von Neumann entropy at saturation with subsystem size for a system of size $N=12$. For $p=0$ this quantity obeys a volume law, but for $p>0$ it saturates to an area law. (b) Scaling of the time $t_{\text {peak }}$ and height $I_{3}^{\max }$ of the intermediate time peak in the tripartite information $I_{3}$, as seen in Fig. 2. The solid lines are power law fits, decaying approximately as $p^{-1}$. With increasing system size, the peak occurs at later times and is smaller in magnitude, indicating that it is (sub)area law.

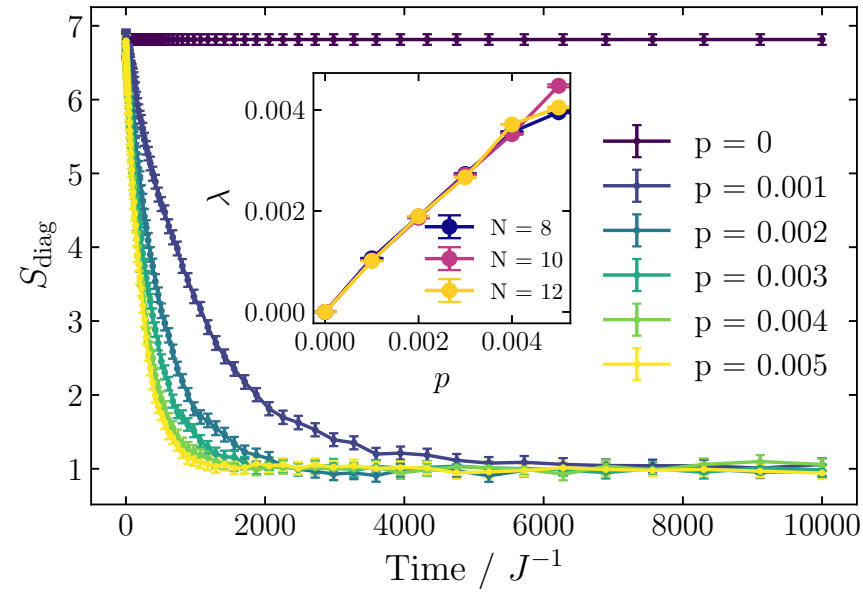

FIG. 4. Dynamics of the diagonal entropy $S_{\text {diag }}$ using the energy eigenbasis, with $Z$-basis measurements and $N=12$. $S_{\text {diag }}$ is preserved by Hamiltonian evolution, so for $p=0$ it remains constant in time. For $p>0$, projective Z-basis measurements cause it to decay exponentially at a rate proportional to $p$. The inset shows the dependence of the early-time decay rate $\lambda$ on $p$ and $N$, with $\lambda \approx p$, independent of $N$.

top panel of Fig. 3(b)]. We expect that if measurements were performed directly in the $l$-bit basis, then this peak would be absent. The fact that our choice of basis differs from the $l$-bit basis in its lack of exponential tails may give rise to this intermediate-time peak.

The inset to Fig. 2 shows that the dynamics can be reasonably well described by the scaling form $-I_{3}(t, p, N)=$ $F\left[t p^{\alpha} / N^{\gamma}\right] / p^{\beta} N^{\delta}$, where $F$ is a scaling function and $\alpha=$ $0.77, \beta=0.80, \gamma=1.14$, and $\delta=2.19$. We also tested scaling $I_{3}$ by an exponential in $N$, so $-I_{3}(t, p, N)=$ $\tilde{F}\left[t p^{\tilde{\alpha}} / N^{\tilde{\gamma}}\right] / p^{\tilde{\beta}} \exp (N \tilde{\delta})$, which resulted in the exponents $\tilde{\alpha}=$ $0.77, \tilde{\beta}=0.77, \tilde{\gamma}=1.14$, and $\tilde{\delta}=0.20$. The quality of both fits were qualitatively similar, so with the system sizes accessible we were unable to rule out power law or exponential scaling of the $I_{3}$ peak height with $N$. Regardless, in either case in the thermodynamic limit $N \rightarrow \infty$ we expect the steadystate tripartite information to scale to zero with $N$ for any $p>0$, indicating the instability of the volume-law phase to measurements.

Focusing further on the transition in the steady state, Fig. 3(a) shows the von Neumann entropy of contiguous subsystems of different sizes in the steady state. Whereas for $p=0$ this quantity is extensive, for $p>0$ it quickly becomes independent of the size of the subsystem, indicating an area law. As discussed in Sec. I, this behavior can be linked to the measurement-induced dynamics of the diagonal entropy $S_{\text {diag }}$, which governs the steady-state entanglement in the measurement-free system. Figure 4 shows that, for any $p>0$, the diagonal entropy decays exponentially in time, before reaching a steady state which is independent of $p$. The inset to Fig. 4 shows that the decay rate $\lambda$ scales approximately as $\lambda \approx p$, independent of system size. We expect this result to hold throughout the fully MBL regime as a result of the strongly localized nature of the $l$-bits and the fact 

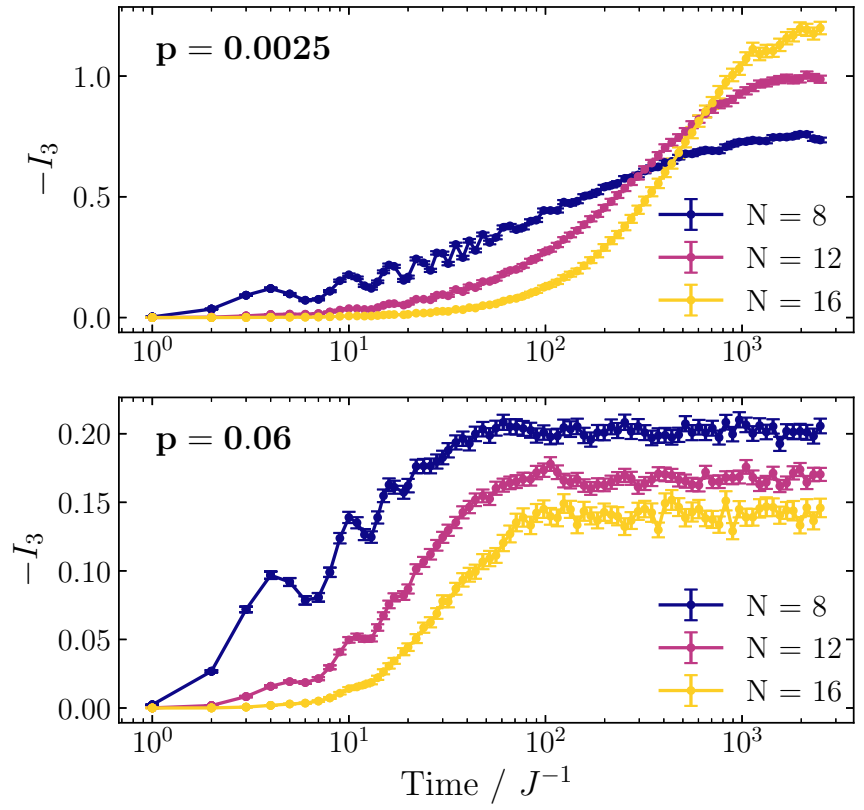

FIG. 5. Comparison of the dynamics of the tripartite information $I_{3}$ with $X$-basis measurements for values of $p$ below and above the transition at $p_{c}^{X} \approx 0.014$. For $p=0.0025, I_{3}$ grows logarithmically in time before saturating to a volume law. By $p=0.06$, the system size scaling has reversed, indicating a transition to an area law.

that the measurements are performed independently on each site.

\section{ENTANGLEMENT DYNAMICS WITH $X$-BASIS MEASUREMENTS}

Having seen in Sec. III the collapse of the MBL steadystate volume law when the dynamics are interspersed with arbitrarily rare $Z$-basis measurements, we now demonstrate that this volume law is more resilient to measurements in the $X$ basis. More precisely, we aim to show that there is a nonzero critical measurement probability $p_{c}^{X}>0$, below which the steady-state volume-law persists. This scenario is similar to the case in previously studied chaotic systems [9-16,18,19,21-25,32,33].

The top panel of Fig. 5 shows the dynamics of the tripartite information $I_{3}$ for nonzero $p=0.0025$, which is below the critical measurement probability $p_{c}^{X} \approx 0.014$. Here the dynamics are qualitatively similar to those for $p=0$, shown in the top panel of Fig. 2, where the magnitude of $I_{3}$ grows logarithmically in time before saturating to a steady-state value which increases with system size. The bottom panel of Fig. 5 shows that, above the transition $p>p_{c}^{X}$, the magnitude of $I_{3}$ still grows logarithmically in time, but no longer saturates to an extensive steady state, indicating a transition to an area law. The growth of the diagonal entropy is qualitatively similar for all nonzero $p$-it follows the functional form $S_{\text {diag }}(t)=S_{\text {diag }}(0) e^{-\lambda t}+S_{\text {diag }}(\infty)\left(1-e^{-\lambda t}\right)$, with the growth rate $\lambda$ proportional to $p$, as shown in Fig. 6. Interestingly, in contrast to the case with Z-basis

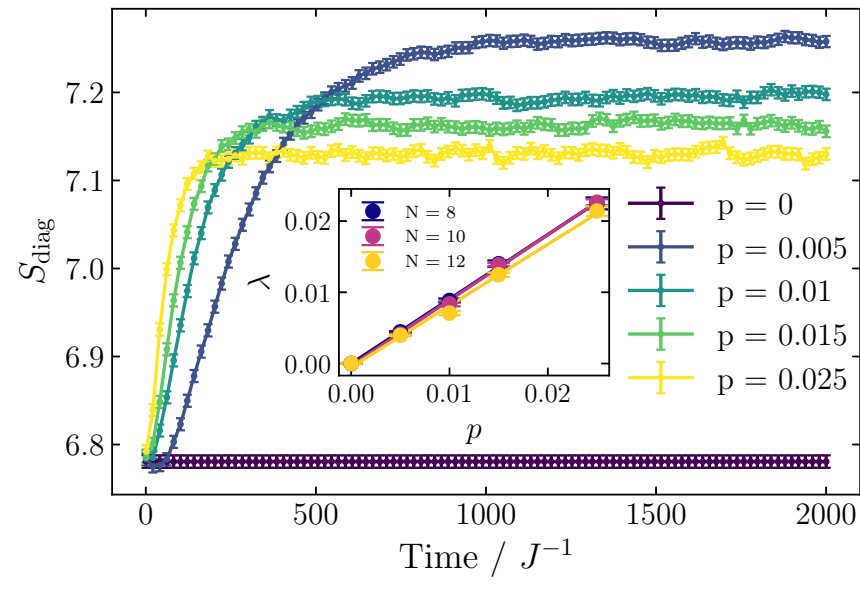

FIG. 6. Dynamics of the diagonal entropy $S_{\text {diag }}$ using the energy eigenbasis, with $X$-basis measurements and $N=12$. $S_{\text {diag }}$ is preserved by Hamiltonian evolution, so for $p=0$ it remains constant in time. For $p>0$, projective $X$-basis measurements cause it to grow as $S_{\text {diag }}(t)=S_{\text {diag }}(0) e^{-\lambda t}+S_{\text {diag }}(\infty)\left(1-e^{-\lambda t}\right)$ at a rate $\lambda$ proportional to $p$. The inset shows the dependence of the early-time decay rate $\lambda$ on $p$ and $N$, with $\lambda \approx p$, independent of $N$.

measurements, here $S_{\text {diag }}(\infty)$ decreases with $p$. This may be a consequence of the finite disorder strength, which means that the energy eigenstates are not exactly product states.

It is also worth noting that, as a result of the increase in diagonal entropy induced by $X$-basis measurements, it is possible to boost the steady-state entanglement relative to $p=0$ for initial states with nonmaximal diagonal entropy, contrary to the usual picture of measurements only destroying entanglement. Of course, this effect is relevant only for fairly small $p$, since for larger $p$ the disentangling power of the individual measurements overcomes the boost from the increased diagonal entropy.

With the goal of studying the transition point between the volume- and area-law phases, we show in Fig. 7 the steadystate tripartite information $I_{3}$ as a function of measurement probability $p$ and system size $N$. To estimate the properties of the critical point, we assume a scaling function of the form $I_{3}(t \rightarrow \infty, p, N)=F\left[\left(p-p_{c}^{X}\right) N^{1 / \nu}\right]$, and perform a fit to minimize the least-squares distance between each scaled point and the line obtained by a linear interpolation between its neighbors (see the Supplemental Material of Ref. [18] for more details). This yields the parameters $p_{c}^{X}=0.014(2)$ and $v=1.3(2)$, where the error bars correspond to the region where the cost function from the fit is less than 1.3 times its minimum. We expect $p_{c}^{X}$ to vary with the time step $\mathrm{d} t$, so we do not believe it will display universal behavior. However, the critical exponent $v$ is close to the value of $v=\frac{4}{3}$ for $2+0 \mathrm{D}$ percolation, similar to the results in random local unitary circuits [18]. To test for the presence of conformal symmetry at the critical point, we also extract the dynamical critical exponent $z$ using the method described in Refs. [15,18] of using the entanglement entropy of a single ancilla qubit as an order parameter for the transition. This yields $z=0.98(4)$, 


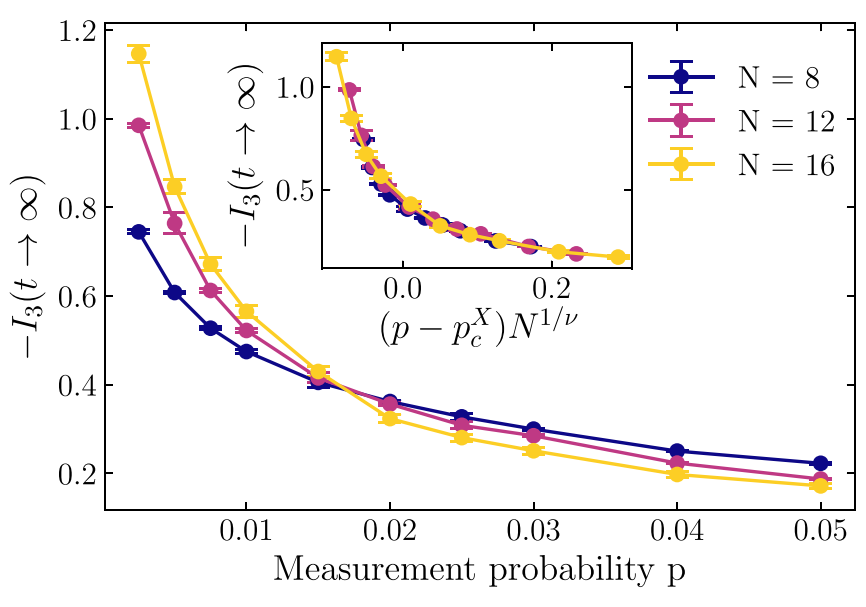

FIG. 7. Steady-state tripartite information $I_{3}$ as a function of measurement probability $p$ and system size $N$. The inset shows a data collapse with the fitted parameters $p_{c}^{X}=0.014(2)$ and $v=1.3(2)$.

as shown in Fig. 8, close to the value of $z=1$ for conformal symmetry.

Finally, having obtained an estimate for the critical point, we examine the scaling of the steady-state entanglement entropy $S_{n}^{l}\left(p=p_{c}^{X}\right)$ with subsystem size $l$ at criticality, where $n$ indicates the Rényi index. In random circuit models with interspersed measurements, $S_{n}^{l}\left(p=p_{c}^{X}\right)$ was found to scale logarithmically with $l$, suggesting an underlying conformal field theory (CFT) description. In Fig. 9(b), we plot $S_{n}^{l}(p=$ $\left.p_{c}^{X}\right)$ as a function of $\ln l$, and find a similar logarithmic scaling, albeit for fairly small subsystem sizes. The coefficient $\alpha(n)$ of the log term depends on the Rényi index $n$, as shown in Fig. 9(c), with the dependence well described

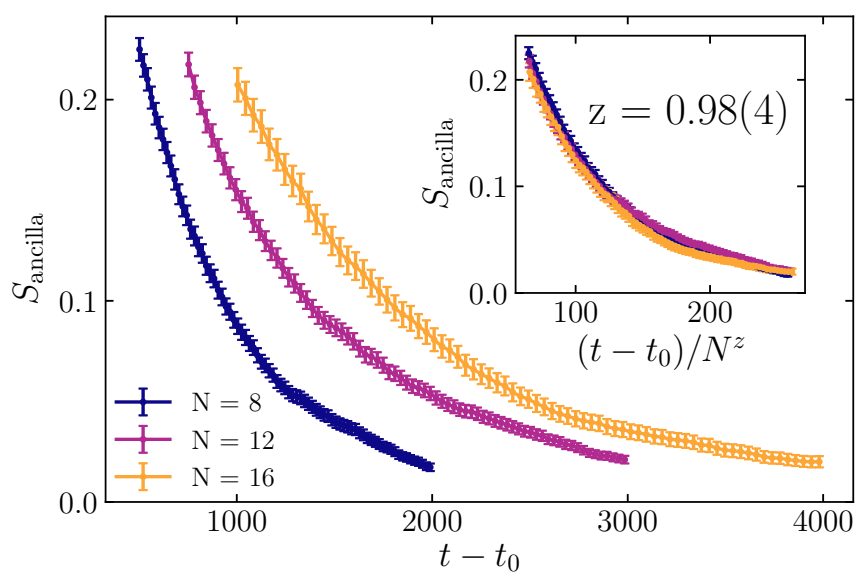

FIG. 8. Extracting the dynamical critical exponent $z=0.98(4)$ using the entanglement entropy of an ancilla qubit as an order parameter for the transition $[15,18]$. The ancilla is maximally entangled with the bulk system at the saturation time $t_{0}$ determined by the dynamics of $I_{3}$, and then we continue with the dynamics described in Sec. II (acting only on the original spins). In performing the data collapse, we exclude times shortly after $t_{0}$ because the scaling only occurs after an intermediate timescale. (a)

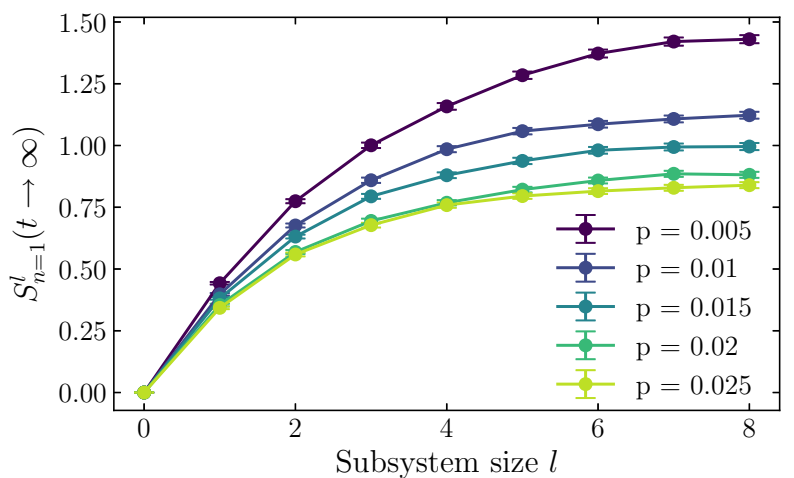

(b)
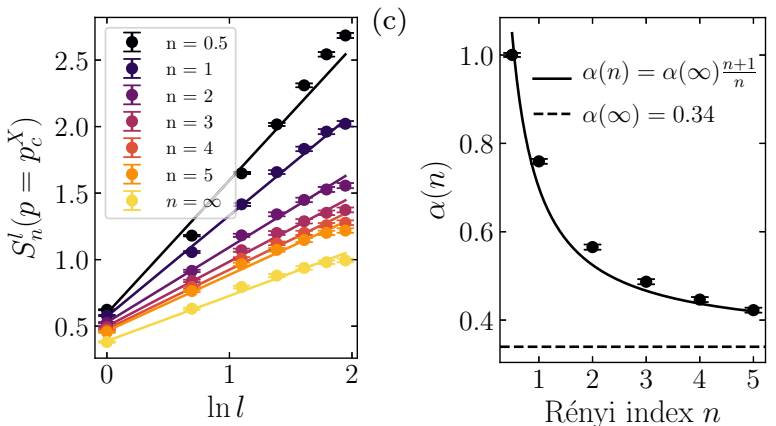

FIG. 9. (a) Scaling of the steady-state von Neumann entropy with subsystem size $l$. The transition from volume to area-law occurs at $p_{c}^{X} \approx 0.014$. (b) The steady-state Rényi- $n$ entropies at criticality as a function of $\ln l$. The straight lines are fits to $S_{n}^{l}\left(p=p_{c}^{X}\right)=$ $\alpha(n) \ln l+\beta(n)$. (c) The dependence of the $\log$ coefficient $\alpha(n)$ on the Rényi index $n$. The solid line is a fit to the form $\alpha(n)=$ $\alpha(\infty)(1+1 / n)$, where $\alpha(\infty) \approx 0.34$.

by $\alpha(n)=\alpha(\infty)(1+1 / n)$, which is the behavior expected for unitary CFTs [68]. Interestingly, in Ref. [18] the authors found for spin- $\frac{1}{2}$ Haar-random circuits that $\alpha(n)$ was not quite described by a fit of this form, but rather required an offset, $\alpha(n)=a(1+1 / n)+b$. This departure from the behavior of unitary CFTs could be seen as consistent with the fact that, in the analytically solvable limit of infinite local Hilbert space dimension $q \rightarrow \infty$, the measurementinduced transition in a Haar-random circuit is described by a nonunitary CFT [13], though there may be important differences between $q \rightarrow \infty$ and finite $q$. Still, that $\alpha(n)$ in this spin- $\frac{1}{2}$ hybrid-MBL system is instead well described by the formula for a unitary CFT suggests that the transition in this system is quite distinct in character from that in Haar-random circuits. Finally, we note in passing that the value of $\alpha(\infty) \approx 0.34$ in this system is far from the value of $\alpha(\infty) \approx 0.49$ observed numerically in spin$\frac{1}{2}$ Haar-random circuits $[18,69]$, but close to the value of $\alpha(n)=\frac{1}{3}$ for all $n \geqslant 1$ predicted in Ref. [13] for the $q \rightarrow$ $\infty$ Haar-random circuit model with periodic boundary conditions, though as emphasized this limit is far from the system considered here. It is also somewhat close to the value of $\ln 2 \times \frac{\sqrt{3}}{\pi} \approx 0.38$ for $2+0 \mathrm{D}$ percolation with periodic boundary conditions [10,69-71], but, at least in hybrid Haarrandom circuits, this value is correct only for the Rényi-0 entropy [13]. 


\section{DISCUSSION}

Measurement-induced entanglement transitions represent an interesting new class of phase transition which shine light on the resilience of quantum entanglement against a classicality-inducing environment. They were initially explored for systems at opposite ends of the spectrum of manybody quantum dynamics: chaotic random unitary circuits and integrable models. In this work we have demonstrated that the nature of the measurement-induced transition in many-body localized (MBL) systems can interpolate between these two extremes, in a way which is consistent with the standard phenomenology of MBL. If the measurements are made in a basis which is scrambled by the MBL dynamics, then the transition from volume- to area-law entanglement occurs at a nonzero measurement probability $p$, similar to previously studied chaotic systems. On the other hand, if the measurements are made in a basis which remains localized by the MBL dynamics, then the volume-law collapses for any nonzero $p$. This distinction does not appear with random unitary circuits, since all local operators are scrambled in time. In MBL systems, the existence of an extensive number of local integrals of motion, the "l-bits," means that not all local operators are scrambled. Instead, only those operators which have low overlap with the $l$-bits are scrambled, and it is for these operators that the volume law will persist for $0 \leqslant p<p_{c}$ if measurements are made in the basis of their eigenstates.

One obvious question is how the measurement-induced entanglement transition (MIT) intersects with the MBL transition. At sufficiently low disorder strength $W<W_{c}$, the Hamiltonian in Eq. (1) is chaotic [41,67], i.e., it satisfies the eigenstate thermalization hypothesis [39,72]. One might expect the MIT in this chaotic Hamiltonian system with shortrange interactions to be in the same universality class as the MIT in random local unitary circuits with the same local Hilbert space dimension. But, from the analysis of the scaling of the critical Rényi entropies in Sec. IV, it appears that the MIT in the MBL phase may be in a distinct universality class to that in Haar-random local unitary circuits. This begs the question of how the conformal field theory describing the MIT critical point changes as one sweeps the disorder strength $W$ across the MBL transition.

It is worth noting that one key difference between the model considered in this paper and random-circuit models is that here there is quenched (spatial) disorder in the unitary part of the dynamics. This is noteworthy because the critical exponent $v=1.3(2)$ we extract violates a naive application of the Harris criterion $v \geqslant 2 / d$ with $d=1[73,74]$. This is despite the fact that we observe $z \approx 1$ at the critical point. We speculate two possible reasons for this violation. The first is that the randomness of the measurements in both space and time means that the overall "disorder" in this problem is no longer quenched, so the Harris criterion may not apply. The second is based on the recent conjecture by $\mathrm{Li}$ et al. [22] that the critical points of these hybrid quantum circuits are described by Euclidean CFTs, where the physical time coordinate essentially acts as a second spatial coordinate. In that sense, the Harris criterion may still apply, but with $d=2$ rather than $d=1$.

We also discussed in Sec. IV how $X$-basis measurements with small but nonzero $p$ can actually increase the steady-state entanglement relative to $p=0$ for initial states with nonmaximal diagonal entropy, as a consequence of the increase in diagonal entropy induced by $X$-basis measurements. This may be somewhat counterintuitive, given the usual picture of measurements destroying entanglement, but suggests the possibility that measurements could be used as a tool to produce states with desirable properties [75], such as high entanglement, in systems where simply evolving with random unitaries is not feasible.

More broadly, there is also the question of how measurements affect the characteristics of phases of pure-unitary dynamics. We have seen, such as in Fig. 5, that at least some of the aspects of MBL phenomenology remain preserved in the volume-law phase even for nonzero $p$, such as the monotonic logarithmic growth of entanglement in time. However, it has also been argued [12] that a steady-state volume law stable to $p>0$ necessarily implies the existence of a subleading logarithmic correction to the volume law, $S(A) \sim \alpha \ln |A|+s|A|$. This has been observed in random Clifford models [16] and appears analytically as an entropic contribution to the free-energy cost of an "entanglement domain wall" [76], so this is one aspect which is qualitatively modified by the presence of measurements. It would be enlightening to see if such a subleading correction is also present in the steady state of this hybrid MBL system, but this is likely out of reach of the small system sizes accessible by exact diagonalization. This may therefore be an opportunity for NISQ-era [77] quantum simulators to probe new physics out of the reach of numerics. Direct measurement of the entanglement entropy associated with a single quantum trajectory may be difficult, owing to the need to perform the exponentially many experimental repetitions associated with the postselection of measurement outcomes [11] and the complexity of measuring an entropy [78,79]. However, there have been proposals for more experimentally feasible probes of the entanglement transition based on the Fisher information [11] and coupled ancilla qubits [15]. It would be interesting to see if these or new techniques could be developed to allow for experimental detection of novel physics, such as the subleading logarithmic corrections to the entanglement entropy, induced by measurements of quantum systems.

\section{ACKNOWLEDGMENTS}

We thank D. Huse and M. Gullans for enlightening discussions on their related work, and especially M. Gullans and A. Nahum for comments on the manuscript. A.P. was funded by the European Research Council (ERC) under the European Union's Horizon 2020 research and innovation programme (Grant agreement No. 853368). O.L. was supported by the Engineering and Physical Sciences Research Council (Grant No. EP/L015242/1). 
[1] M. Schlosshauer, Decoherence, the measurement problem, and interpretations of quantum mechanics, Rev. Mod. Phys. 76, 1267 (2005).

[2] C. H. Bennett, G. Brassard, S. Popescu, B. Schumacher, J. A. Smolin, and W. K. Wootters, Purification of Noisy Entanglement and Faithful Teleportation Via Noisy Channels, Phys. Rev. Lett. 76, 722 (1996).

[3] S. Bravyi and A. Kitaev, Universal quantum computation with ideal Clifford gates and noisy ancillas, Phys. Rev. A 71, 022316 (2005).

[4] C. H. Bennett, D. P. DiVincenzo, J. A. Smolin, and W. K. Wootters, Mixed-state entanglement and quantum error correction, Phys. Rev. A 54, 3824 (1996).

[5] R. Vijay, D. H. Slichter, and I. Siddiqi, Observation of Quantum Jumps in a Superconducting Artificial Atom, Phys. Rev. Lett. 106, 110502 (2011).

[6] K. W. Murch, S. J. Weber, C. Macklin, and I. Siddiqi, Observing single quantum trajectories of a superconducting quantum bit, Nature (London) 502, 211 (2013).

[7] N. Ofek, A. Petrenko, R. Heeres, P. Reinhold, Z. Leghtas, B. Vlastakis, Y. Liu, L. Frunzio, S. M. Girvin, L. Jiang, M. Mirrahimi, M. H. Devoret, and R. J. Schoelkopf, Extending the lifetime of a quantum bit with error correction in superconducting circuits, Nature (London) 536, 441 (2016).

[8] Z. Minev, S. Mundhada, S. Shankar, P. Reinhold, R. GutiérrezJáuregui, R. Schoelkopf, M. Mirrahimi, H. Carmichael, and M. Devoret, To catch and reverse a quantum jump mid-flight, Nature (London) 570, 200 (2019).

[9] Y. Li, X. Chen, and M. P. A. Fisher, Quantum Zeno effect and the many-body entanglement transition, Phys. Rev. B 98, 205136 (2018).

[10] B. Skinner, J. Ruhman, and A. Nahum, Measurement-Induced Phase Transitions in the Dynamics of Entanglement, Phys. Rev. X 9, 031009 (2019).

[11] Y. Bao, S. Choi, and E. Altman, Theory of the phase transition in random unitary circuits with measurements, Phys. Rev. B 101, 104301 (2020).

[12] A. Chan, R. M. Nandkishore, M. Pretko, and G. Smith, Unitaryprojective entanglement dynamics, Phys. Rev. B 99, 224307 (2019).

[13] C.-M. Jian, Y.-Z. You, R. Vasseur, and A. W. W. Ludwig, Measurement-induced criticality in random quantum circuits, Phys. Rev. B 101, 104302 (2020).

[14] M. Szyniszewski, A. Romito, and H. Schomerus, Entanglement transition from variable-strength weak measurements, Phys. Rev. B 100, 064204 (2019).

[15] M. J. Gullans and D. A. Huse, Scalable Probes of Measurement-Induced Criticality, Phys. Rev. Lett. 125, 070606 (2020).

[16] Y. Li, X. Chen, and M. P. A. Fisher, Measurement-driven entanglement transition in hybrid quantum circuits, Phys. Rev. B 100, 134306 (2019).

[17] X. Cao, A. Tilloy, and A. De Luca, Entanglement in a fermion chain under continuous monitoring, SciPost Phys. 7, 024 (2019).

[18] A. Zabalo, M. J. Gullans, J. H. Wilson, S. Gopalakrishnan, D. A. Huse, and J. H. Pixley, Critical properties of the measurementinduced transition in random quantum circuits, Phys. Rev. B 101, 060301(R) (2020).
[19] Q. Tang and W. Zhu, Measurement-induced phase transition: A case study in the nonintegrable model by density-matrix renormalization group calculations, Phys. Rev. Research 2, 013022 (2020).

[20] D. Rossini and E. Vicari, Measurement-induced dynamics of many-body systems at quantum criticality, Phys. Rev. B 102, 035119 (2020).

[21] S. Goto and I. Danshita, Measurement-induced transitions of the entanglement scaling law in ultracold gases with controllable dissipation, Phys. Rev. A 102, 033316 (2020).

[22] Y. Li, X. Chen, A. W. W. Ludwig, and M. P. A. Fisher, Conformal invariance and quantum non-locality in hybrid quantum circuits, arXiv:2003.12721.

[23] J. Lopez-Piqueres, B. Ware, and R. Vasseur, Mean-field theory of entanglement transitions from random tree tensor networks, Phys. Rev. B 102, 064202 (2020).

[24] R. Fan, S. Vijay, A. Vishwanath, and Y.-Z. You, Self-organized error correction in random unitary circuits with measurement, arXiv:2002.12385.

[25] L. Zhang, J. A. Reyes, S. Kourtis, C. Chamon, E. R. Mucciolo, and A. E. Ruckenstein, Nonuniversal entanglement level statistics in projection-driven quantum circuits, Phys. Rev. B 101, 235104 (2020).

[26] M. Szyniszewski, A. Romito, and H. Schomerus, Universality of entanglement transitions from stroboscopic to continuous measurements, arXiv:2005.01863.

[27] Y. Fuji and Y. Ashida, Measurement-induced quantum criticality under continuous monitoring, Phys. Rev. B 102, 054302 (2020).

[28] S. Sang and T. H. Hsieh, Measurement protected quantum phases, arXiv:2004.09509.

[29] A. Lavasani, Y. Alavirad, and M. Barkeshli, Measurementinduced topological entanglement transitions in symmetric random quantum circuits, arXiv:2004.07243.

[30] O. Shtanko, Y. A. Kharkov, L. P. García-Pintos, and A. V. Gorshkov, Classical models of entanglement in monitored random circuits, arXiv:2004.06736.

[31] S. Vijay, Measurement-driven phase transition within a volumelaw entangled phase, arXiv:2005.03052.

[32] S. Choi, Y. Bao, X.-L. Qi, and E. Altman, Quantum Error Correction and Entanglement Phase Transition in Random Unitary Circuits with Projective Measurements, Phys. Rev. Lett. 125, 030505 (2020).

[33] M. J. Gullans and D. A. Huse, Dynamical purification phase transition induced by quantum measurements, arXiv:1905.05195.

[34] J. Napp, R. L. La Placa, A. M. Dalzell, F. G. S. L. Brandao, and A. W. Harrow, Efficient classical simulation of random shallow 2D quantum circuits, arXiv:2001.00021.

[35] O. Mandel, M. Greiner, A. Widera, T. Rom, T. W. Hänsch, and I. Bloch, Controlled collisions for multi-particle entanglement of optically trapped atoms, Nature (London) 425, 937 (2003).

[36] A. M. Kaufman, M. E. Tai, A. Lukin, M. Rispoli, R. Schittko, P. M. Preiss, and M. Greiner, Quantum thermalization through entanglement in an isolated many-body system, Science 353, 794 (2016)

[37] H. Bernien, S. Schwartz, A. Keesling, H. Levine, A. Omran, H. Pichler, S. Choi, A. S. Zibrov, M. Endres, M. Greiner et al., 
Probing many-body dynamics on a 51-atom quantum simulator, Nature (London) 551, 579 (2017).

[38] J. Zhang, G. Pagano, P. W. Hess, A. Kyprianidis, P. Becker, H. Kaplan, A. V. Gorshkov, Z.-X. Gong, and C. Monroe, Observation of a many-body dynamical phase transition with a 53-qubit quantum simulator, Nature (London) 551, 601 (2017).

[39] L. D’Alessio, Y. Kafri, A. Polkovnikov, and M. Rigol, From quantum chaos and eigenstate thermalization to statistical mechanics and thermodynamics, Adv. Phys. 65, 239 (2016).

[40] D. M. Basko, I. L. Aleiner, and B. L. Altshuler, Metal-insulator transition in a weakly interacting many-electron system with localized single-particle states, Ann. Phys. (NY) 321, 1126 (2006).

[41] A. Pal and D. A. Huse, Many-body localization phase transition, Phys. Rev. B 82, 174411 (2010).

[42] V. Oganesyan and D. A. Huse, Localization of interacting fermions at high temperature, Phys. Rev. B 75, 155111 (2007).

[43] R. Nandkishore and D. A. Huse, Many-body localization and thermalization in quantum statistical mechanics, Annu. Rev. Condens. Matter Phys. 6, 15 (2015).

[44] D. A. Abanin, E. Altman, I. Bloch, and M. Serbyn, Many-body localization, thermalization, and entanglement, Rev. Mod. Phys. 91, 021001 (2019).

[45] D. A. Abanin and Z. Papić, Recent progress in many-body localization, Ann. Phys. (Leipzig) 529, 1700169 (2017).

[46] S. A. Parameswaran and R. Vasseur, Many-body localization, symmetry and topology, Rep. Prog. Phys. 81, 082501 (2018).

[47] M. Žnidarič, T. Prosen, and P. Prelovšek, Many-body localization in the Heisenberg $x x z$ magnet in a random field, Phys. Rev. B 77, 064426 (2008).

[48] J. H. Bardarson, F. Pollmann, and J. E. Moore, Unbounded Growth of Entanglement in Models of Many-Body Localization, Phys. Rev. Lett. 109, 017202 (2012).

[49] D. A. Huse, R. Nandkishore, and V. Oganesyan, Phenomenology of fully many-body-localized systems, Phys. Rev. B 90, 174202 (2014).

[50] M. Serbyn, Z. Papić, and D. A. Abanin, Universal Slow Growth of Entanglement in Interacting Strongly Disordered Systems, Phys. Rev. Lett. 110, 260601 (2013).

[51] A. Nanduri, H. Kim, and D. A. Huse, Entanglement spreading in a many-body localized system, Phys. Rev. B 90, 064201 (2014).

[52] J. Z. Imbrie, On many-body localization for quantum spin chains, J. Stat. Phys. 163, 998 (2016).

[53] V. Ros, M. Müller, and A. Scardicchio, Integrals of motion in the many-body localized phase, Nucl. Phys. B 891, 420 (2015).

[54] Y. Huang, Y.-L. Zhang, and X. Chen, Out-of-time-ordered correlators in many-body localized systems, Ann. Phys. (Leipzig) 529, 1600318 (2017).

[55] R.-Q. He and Z.-Y. Lu, Characterizing many-body localization by out-of-time-ordered correlation, Phys. Rev. B 95, 054201 (2017).

[56] B. Swingle and D. Chowdhury, Slow scrambling in disordered quantum systems, Phys. Rev. B 95, 060201(R) (2017).

[57] X. Chen, T. Zhou, D. A. Huse, and E. Fradkin, Out-of-timeorder correlations in many-body localized and thermal phases, Ann. Phys. (Leipzig) 529, 1600332 (2017).
[58] R. Fan, P. Zhang, H. Shen, and H. Zhai, Out-of-time-order correlation for many-body localization, Sci. Bull. 62, 707 (2017).

[59] I. MacCormack, M. T. Tan, J. Kudler-Flam, and S. Ryu, Operator and entanglement growth in non-thermalizing systems: Many-body localization and the random singlet phase, arXiv:2001.08222.

[60] A. Chandran, I. H. Kim, G. Vidal, and D. A. Abanin, Constructing local integrals of motion in the many-body localized phase, Phys. Rev. B 91, 085425 (2015).

[61] L. Rademaker and M. Ortuño, Explicit Local Integrals of Motion for the Many-Body Localized State, Phys. Rev. Lett. 116, 010404 (2016).

[62] D. Pekker, B. K. Clark, V. Oganesyan, and G. Refael, Fixed Points of Wegner-Wilson Flows and Many-Body Localization, Phys. Rev. Lett. 119, 075701 (2017).

[63] A. K. Kulshreshtha, A. Pal, T. B. Wahl, and S. H. Simon, Behavior of 1-bits near the many-body localization transition, Phys. Rev. B 98, 184201 (2018).

[64] M. Goihl, M. Gluza, C. Krumnow, and J. Eisert, Construction of exact constants of motion and effective models for many-body localized systems, Phys. Rev. B 97, 134202 (2018).

[65] M. Ippoliti, M. J. Gullans, S. Gopalakrishnan, D. A. Huse, and V. Khemani, Entanglement phase transitions in measurementonly dynamics, arXiv:2004.09560.

[66] D. J. Luitz, N. Laflorencie, and F. Alet, Many-body localization edge in the random-field Heisenberg chain, Phys. Rev. B 91, 081103(R) (2015).

[67] E. V. H. Doggen, F. Schindler, K. S. Tikhonov, A. D. Mirlin, T. Neupert, D. G. Polyakov, and I. V. Gornyi, Many-body localization and delocalization in large quantum chains, Phys. Rev. B 98, 174202 (2018).

[68] P. Calabrese and A. Lefevre, Entanglement spectrum in one-dimensional systems, Phys. Rev. A 78, 032329 (2008).

[69] Note that Zabalo et al. and Skinner et al. measure their entropies with base-2 logs, whereas we use natural logs, so one has to multiply their values by $\ln 2$ to compare.

[70] J. Cardy, Linking Numbers for Self-Avoiding Loops and Percolation: Application to the Spin Quantum Hall Transition, Phys. Rev. Lett. 84, 3507 (2000).

[71] J. Jiang and C.-L. Yao, Critical first-passage percolation starting on the boundary, Stochast. Process. Appl. 129, 2049 (2019).

[72] M. Srednicki, Chaos and quantum thermalization, Phys. Rev. E 50, 888 (1994).

[73] A. B. Harris, Effect of random defects on the critical behavior of Ising models, J. Phys. C: Solid State Phys. 7, 1671 (1974).

[74] J. T. Chayes, L. Chayes, D. S. Fisher, and T. Spencer, Correlation length bounds for disordered Ising ferromagnets, Commun. Math. Phys. 120, 501 (1989).

[75] S. Roy, J. T. Chalker, I. V. Gornyi, and Y. Gefen, Measurementinduced steering of quantum systems, Phys. Rev. Res. 2, 033347 (2020).

[76] Y. Li and M. P. A. Fisher, Statistical Mechanics of Quantum Error-Correcting Codes, arXiv:2007.03822. 
[77] J. Preskill, Quantum Computing in the NISQ era and beyond, Quantum 2, 79 (2018).

[78] R. O'Donnell and J. Wright, Quantum Spectrum Testing, in Proceedings of the Forty-Seventh Annual ACM Symposium on Theory of Computing, STOC '15 (Asso- ciation for Computing Machinery, Portland, OR, 2015), pp. 529-538.

[79] T. $\mathrm{Li}$ and $\mathrm{X}$. Wu, Quantum query complexity of entropy estimation, IEEE Trans. Inf. Theory 65, 2899 (2019). 